Int. J. Dev. Biol. 53: 177-185 (2009)

doi: $10.1387 / \mathrm{ijdb} .072488 \mathrm{mb}$

\title{
Arabidopsis monomeric G-proteins, markers of early and late events in cell differentiation
}

\author{
MARIETTE BEDHOMME*, CHANTAL MATHIEU, AMADA PULIDO, YVES HENRY\# \\ and CATHERINE BERGOUNIOUX\# \\ Institut de Biotechnologie des Plantes, UMR CNRS 8618, Orsay, France
}

\begin{abstract}
In Schizosaccharomyces pombe, septum formation is intricately controlled by proteins which constitute the SIN (Septum Initiation Network) signalling cascade. The SIN ensures the coordination between mitotic exit and cytokinesis. Yeast spg1p is a core component of the SIN pathway and we have previously characterized the two orthologs of this G-protein in Arabidopsis thaliana (named AtSGP1 and 2). In this work, the cell and tissue expression of AtSGPgenes during plant development has been analysed using AtSGP promoter::GUS fusions in stably transformed A. thaliana lines. AtSGP1 promoter activity was restricted to the quiescent centre, collumella cells, stomata guard cells and the stele while AtSGP2 promoter activity was detected in atrichoblasts, trichomes and pollen. The observed promoter activities are in accordance with publicly available pollen, stomata guard cell and root transcriptome data. Two-hybrid experiments previously evidenced an interaction between AtMAP3Kepsilon1 and AtSGP1. The AtMAP3Kepsilon1 promoter activity was detected in root apices, trichomes and ovule integuments. A genetic approach involving both markers of these specialized cells and mutant backgrounds was used to reinforce our hypothesis. It appears that, although highly conserved between plants and fungi, the spg1p G-protein has evolved in plants to perform a function different from the SIN pathway. Interestingly, cells expressing AtSGPs possessed limited or null mitotic activity. Our data suggests that AtSGP are crucial signalling components involved either in early cell fate specification, or in the final steps of cell differentiation. This is an interesting starting point for a wider study devoted to functional experiments designed to test these hypotheses.
\end{abstract}

KEY WORDS: Arabidopsis thaliana, AtSGPs, atrichoblasts, pollen, quiescent centre

\section{Introduction}

The development and growth of plant organisms is regulated by a series of intricate signalling pathways and transcriptional networks. They act to specify cell types and to maintain cell differentiation. During plant development, the implementation of the dynamic processes of cell division requires the integration and evaluation of signals coming from diverse cellular programs. Development integrates highly complex mechanisms. Cell specification in plants is determined through two different systems shared by animals and plants, cell position and cell lineage. The first one uses positional information, meaning that the development of a particular cell type is determined by its localisation relative to another cell type (Dolan, 2006). The establishment of a new cell type during development also uses a mechanism based on the control of an initial asymmetric cell division to drive either cell position or cell lineage (MacAlister et al., 2007). To date, only a single signalling element, the receptor-like kinase SCRAMBLED, has been shown to function in cell type specification.

Previously, we identified and characterized several signalling components (kinases, G-proteins) in Arabidopsis thaliana that were closely related to the core components of the SIN (Septum Initiation Network) pathway of Schizosaccharomyces pombe. The SIN pathway controls events at the end of mitosis, coordinating mitotic exit and cytokinesis (Simanis, 2003). The G-protein

Abbreviations used in this paper: AtSGP, Arabdiposis thaliana G-protein; SIN, septum initiation network; QC, quiescent centre.

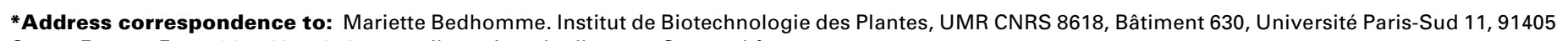
Orsay, France. Fax: +33-1-6915-3424. e-mail: mariette.bedhomme@u-psud.fr

\#Note: Y. Henry and C. Bergounioux have contributed equally to this work. 
AtSGP and the kinase AtMAP3Kepsilon are orthologues of the core components spg1 and $c d c 7$ of the yeast SIN pathway (Champion et al., 2004; Jouannic et al., 2001). An original feature is that each of these SIN components possesses two paralogues in the $A$. thaliana genome, namely AtSGP1 - AtSGP2 and AtMAP3Kepsilon 1- AtMAP3Kepsilon2. Overexpression of the $A$. thaliana genes AtSGP1 and AtSGP2 in yeast cells induced multiple rounds of septum formation without cell cleavage, similarly to their orthologue spg1 (Champion et al., 2004).

In $S$. pombe, spg1p and cdc7p interact both genetically and physically (Schmidt et al., 1997). Assays in yeast showed an interaction between cdc7p and both AtSGP1 and AtSGP2. On the other hand no interaction could be detected between Brassica napusMAP3Kepsilon1 and spg1p, while BnMAP3Kepsilon1 could interact with AtSGP1, but this interaction was not detected with AtSGP2 (Champion et al., 2004), a finding which supports the possibility of a functional difference.

Our previous results also revealed that, depending on the organs, AtSGP1 showed higher expression (root) or reduced expression (flower) compared to its paralogue AtSGP2(Champion et al., 2004). In this work, we further examined the possibility of AtSGP involvement in cell division or in cytokinesis, by carefully analysing the localization of AtSGP1 and $A T S G P 2$ promoter activity using promoter:: GUS constructs. Expression studies were performed in the reference Columbia ecotype and in various mutant backgrounds related to the cell types expressing each AtSGPgenes. AtSGP1 and $A T S G P 2$ promoter activity was observed in specialized cell types, with limited or null mitotic activity, including epidermal cells (atrichoblasts, trichomes, stomata guard cells), mature pollen, and the quiescent centre. Our data suggest that these GTPases act in early and late differentiation signalling mechanisms in several cell types.

\section{Results}

\section{AtSGP1 promoter activity in particu- lar cell types}

Using transgenic Arabidopsis thaliana plants stably transformed with the AtSGP1promoter:: $\beta$-glucuronidase (GUS) fusion construct, cell- and tissue-specific activity of the AtSGP1promoter was examined in planta. However, despite the previously reported AtSGP1 expression at G2-M phases (Champion et al., 2004), GUS staining appeared neither in the shoot apical meristem nor in the root meristematic zone containing mitotically active cells. In flowers, AtSGP1 promoter activity was detected in the sepals, the style and the connective tissue of anthers (Fig. 1A). Furthermore, AtSGP1 promoter activity was observed in very specialized leaf cells, the stomata guard cells (Fig. 1C), but GUS coloration was not seen at an earlier developmental stage in guard cell mother cells, nor in the meristemoid cells. AtSGP1 promoter activity was found in stomata guard cells on both the abaxial and adaxial leaf epidermis. In addition, GUS staining was detected in young leaf hydathodes (Fig. 1B). Hydathodes are often found at the leaf margin, at the end of vascular bundles. They are specialised structures involved in secretion or water exudation.

Further histochemical analysis using ten day-old seedlings showed that the AtSGP1 promoter directed expression of the GUS reporter gene particularly in the root tip of both primary and lateral roots. Indeed, the AtSGP1 promoter.:GUS fusion conferred an important expression in the QC cells (Fig. 1E). The QC consists of a four-cell organizing center that maintains stem
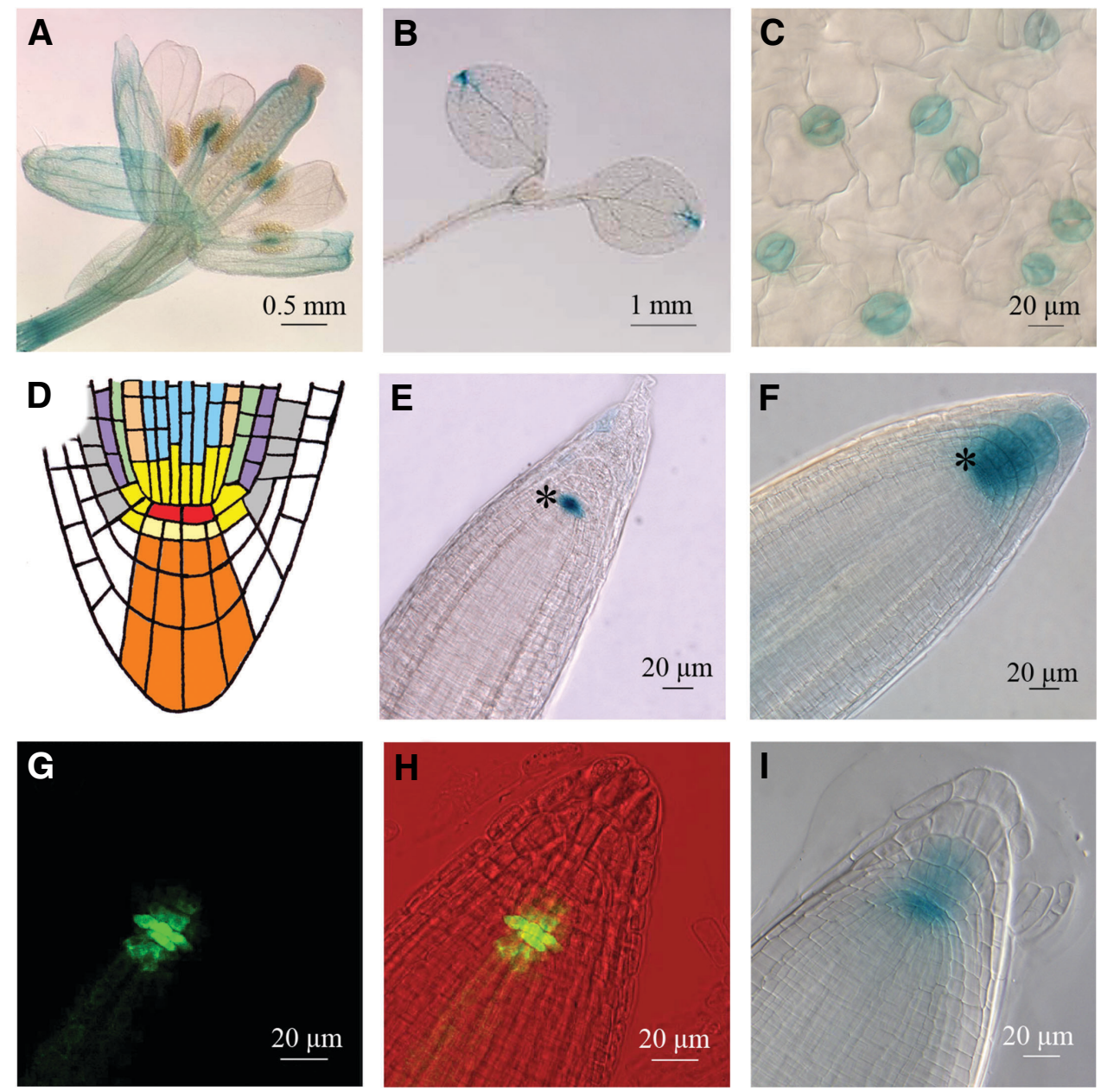

Fig. 1. Arabidopsis thaliana AtSGP1 promoter activity patterns in transgenic $\mathrm{T}_{\mathbf{2}}$ lines. The promoter activity is visualised by GUS staining of flower (A), 10 days old seedling (B) and leaf epidermis (C). Note the staining in connectives (A), hydathodes (B) and stomata guard cells (C). (D) Colorized drawing of the root apical meristem region: gray: epidermis, purple: cortex, green: endodermis, peach: pericycle, blue: stele, white: lateral root cap, orange: columella, yellow: initials (stem cells), red: quiescent centre (QC). (E,F) GUS activity in the root apex of 12 day old seedlings. Depending on the different lines (e,f) GUS activity was restricted to the QC (marked with an asterisks $\left.{ }^{*}\right)$, or observed in $Q C$, stem cells and columella cells. $(\mathbf{G}, \mathbf{H}, \mathbf{I})$ AtSGP1 promoter activity in DR5::GFP lines. Both gene reporter activities colocalize at the $Q C$. 

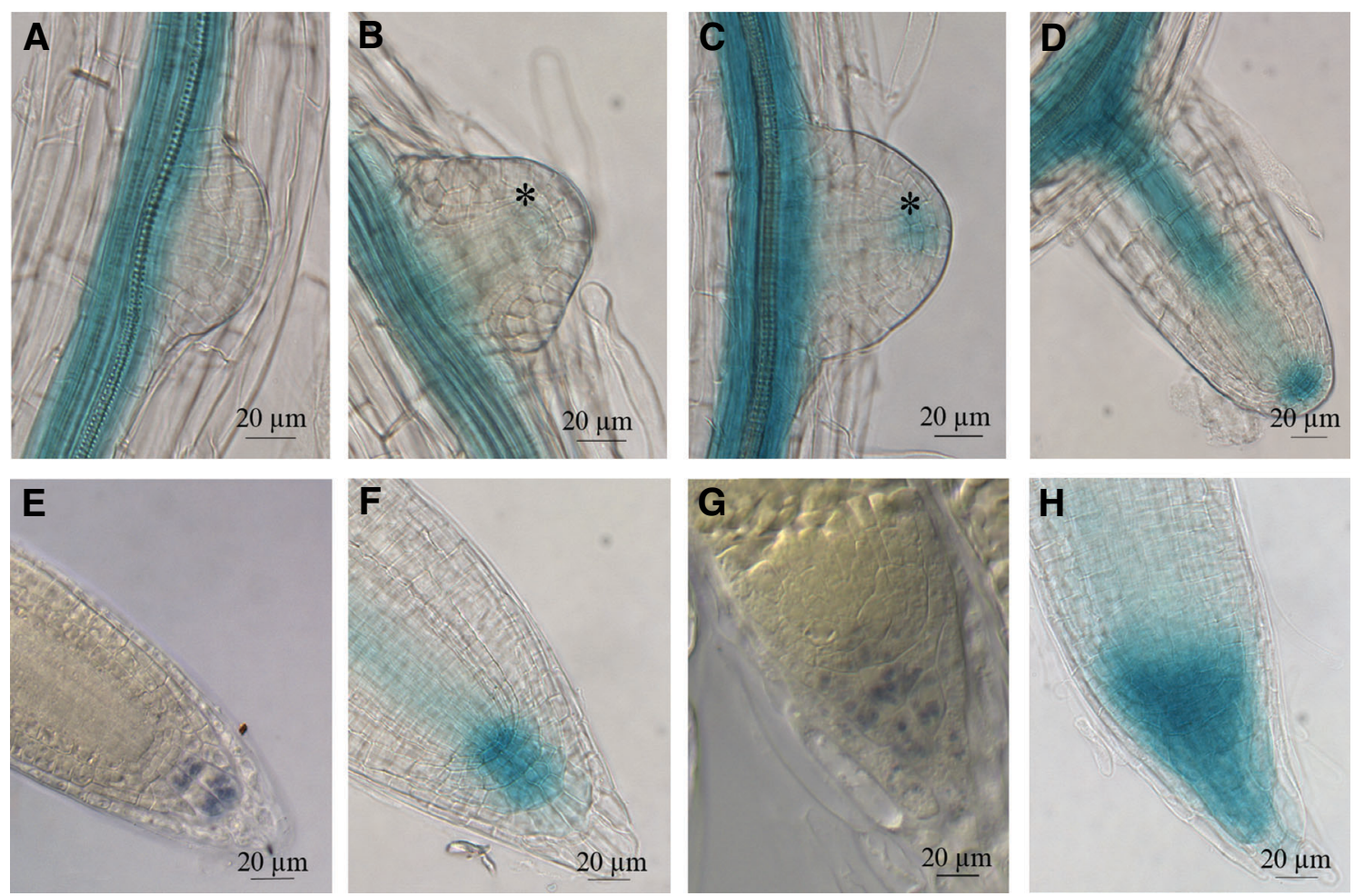

Fig. 2. AtSGP1 promoter activity correlates with the positioning of the quiescent centre (QC). (A,D) During initiation and development of the lateral root. QC is marked with an asterisk. AtSGP1 promoter activity is expanded in the plt1 plt2 double mutant background (G,H) compared to the wild type (E,F). Starch granule staining marks differentiated columella cells $(\mathbf{E}, \mathbf{G})$.

cells mitotically active. Together the QC and the stem cells constitute the stem cell niche (Fig. 1D) (van den Berg et al., 1997). A weaker expression was observed in cells (i.e. the columella initials, ground tissue or columella root cap) proximal to the QC, in most of the 20 lines tested (Fig. 1F). In order to ascertain the activity of the AtSGP1 promoter in the QC, a plant line was generated that possessed both AtSGP1promoter::GUS and the auxin distribution marker DR5::GFP. Both reporter genes were assayed in the same roots. We first observed GFP fluorescence driven by the DR5 promoter in the QC (Fig. 1G). Then, a histochemical analysis performed on the same root tips showed that GUS-expressing cells overlapped with the GFPexpressing cells (Fig. $1 \mathrm{H}, \mathrm{I}$ ). The co-localization of the AtSGP1 promoter activity (ie GUS staining) with the DR5::GFP reporter activation confirmed that $A t S G P 1$ promoter activity was present in the QC, and thus associated with high auxin concentrations (Ulmasov et al., 1997).

GUS staining was monitored during lateral root initiation and development in order to determine at what stage the AtSGP1 promoter activity was induced. No promoter activity was detected in early lateral root primordia emerging from pericycle cells (Fig. 2A). At further developmental stages, GUS staining was associated only with QC cells (Fig. 2 B,C,D). This suggests AtSGP1 promoter activity to be associated with QC positioning.

To determine whether or not AtSGP1 gene expression depended on QC-specific genes, we studied putative genetic interactions with PLETHORA1 (PLT1) and PLETHORA2 (PLT2) genes. Both $P L T$ genes are required for specification and maintenance of the $\mathrm{QC}$ and stem cells in the root meristem
(Aida et al., 2004). In a p/t1-4 plt2-2 double mutant (mentioned thereafter as $p / t 1$ pltz), roots do not possess a functional QC, the columella contains an increased number of differentiated cells and its stratified structure is disturbed. Indeed, starch granules accumulated in all columella layers (compare Fig. 2 $E, G$ ), including cells at the position of the stem cells (Aida et al., 2004). The root apical meristem is strongly modified in the plt 1 p/t2 double mutant. We analysed the effect of the p/t1 p/t2 mutations on AtSGP1 promoter activity by introducing the AtSGP1promoter::GUS construct into the plt1 p/t2 double mutant. In the root meristem of $\mathrm{T}_{2}$ transformed $p / t 1$ p/t2 lines, GUS staining expanded to at least two additional layers of differentiated columella cells containing starch granules, proximal to the mis-specified QC cells (compare Fig. 2 F,H). AtSGP1 promoter activity was not suppressed in the plt1 plt2 double mutant background, thus showing that PLETHORA is not required for AtSGP1 promoter activity. Nevertheless, in the absence of PLETHORA expression both the QC fate and the initial cell fate are not specified, and the root meristem contains much more differentiated cells. These changes lead to a significantly enlarged AtSGP1 promoter activity in the root meristem. Therefore, the expression domain of AtSGP1 promoter activity depends on the PLETHORA genes.

About twenty transformed lines were found to exhibit a GUS expression pattern along root vascular tissues with weaker expression in mitotically active cells. Figure 2 A,B,C,D,F reveals AtSGP1 promoter activity in the stele, at stages including differentiated pericycle and xylem cells. Weak staining was also detected in the stele at earlier stages upstream of the QC 
(Fig. 2 D,F). Our data suggest a putative role for AtSGP1 in the differentiation of different stele cell types.

\section{AtSGP2 promoter activity is also detected in specialised cells}

Histochemical staining of the primary root of 10day-old transgenic plants was performed on $\mathrm{T} 1$ and T2 plants transformed with a AtSGP2promoter::GUS construct. The localisation of GUS activity was totally different from that obtained with the promoter of its paralogue AtSGP1. It appeared neither in the apical meristem, nor in the QC. AtSGP2 promoter activity in epidermal cells gradually increased in the upstream part of the division zone. The GUS staining was detected in differentiating cells near the onset of cell elongation (Fig. 3A). The staining was not homogenous, as files of stained cells were separated by files of unstained cells (Fig. $3 \mathrm{~A}, \mathrm{~B}$ ). The AtSGP2promoter activity was never observed in cell files within the maturation zone. To determine more precisely the location of stained cells, transverse sections were performed from stained and embedded elongation zones of primary roots. The blue staining was limited to those epidermal cells located over tangential cortical cell walls (i.e. at the position of differentiating atrichoblast cells) (Fig. 3B insert), while no staining was detected in epidermal cells located over the junction of cortical cells, at the position of the differentiating trichoblasts (i.e. hair cells).

To determine whether or not the AtSGP2 promoter activity is required to ensure that cells located in particular positions of the $A$. thaliana root epidermis differentiate into atrichoblast epidermal cells, the AtSGP2 promoter activity was examined in a mutant background. In the SCRAMBLED (SCM) mutant scm-2, the GLABRA2promoter activity displayed a patchy distribution in the root epidermis (Kwak et al., 2005). We transformed the scm-2 mutant with the AtSGP2 promoter:: GUS construct and we analysed roots from ten $\mathrm{T}_{2}$ resistant lines. In the scm-2mutant background, the pattern of AtSGP2 promoter activity was disturbed. Stained cells were observed in all cell files, in an identical manner to GLABRA2 promoter activity in the scm-2 mutant, but with a weak blue staining (Fig. 3C). This finding suggests that the $S C M$ receptor kinase gene is epistatic to AtSGP2.

In leaves, expression of the AtSGP2promoter was confined to trichomes. During leaf development, GUS activity was found in trichome cells at different developmental stages, from epidermal outgrowth to branch initiation (Fig. 3D) and cell maturation (Fig. 3 E,F). However, the socket cells, arranged around the basal part of the mature trichome, remained unstained (Fig. 3F).

The previously detected high AtSGP2 transcript levels in flower buds and mature flowers (Champion etal., 2004), prompted us to further explore AtSGP2 expression in the different flower organs. At early stages of flower development, in flowers from
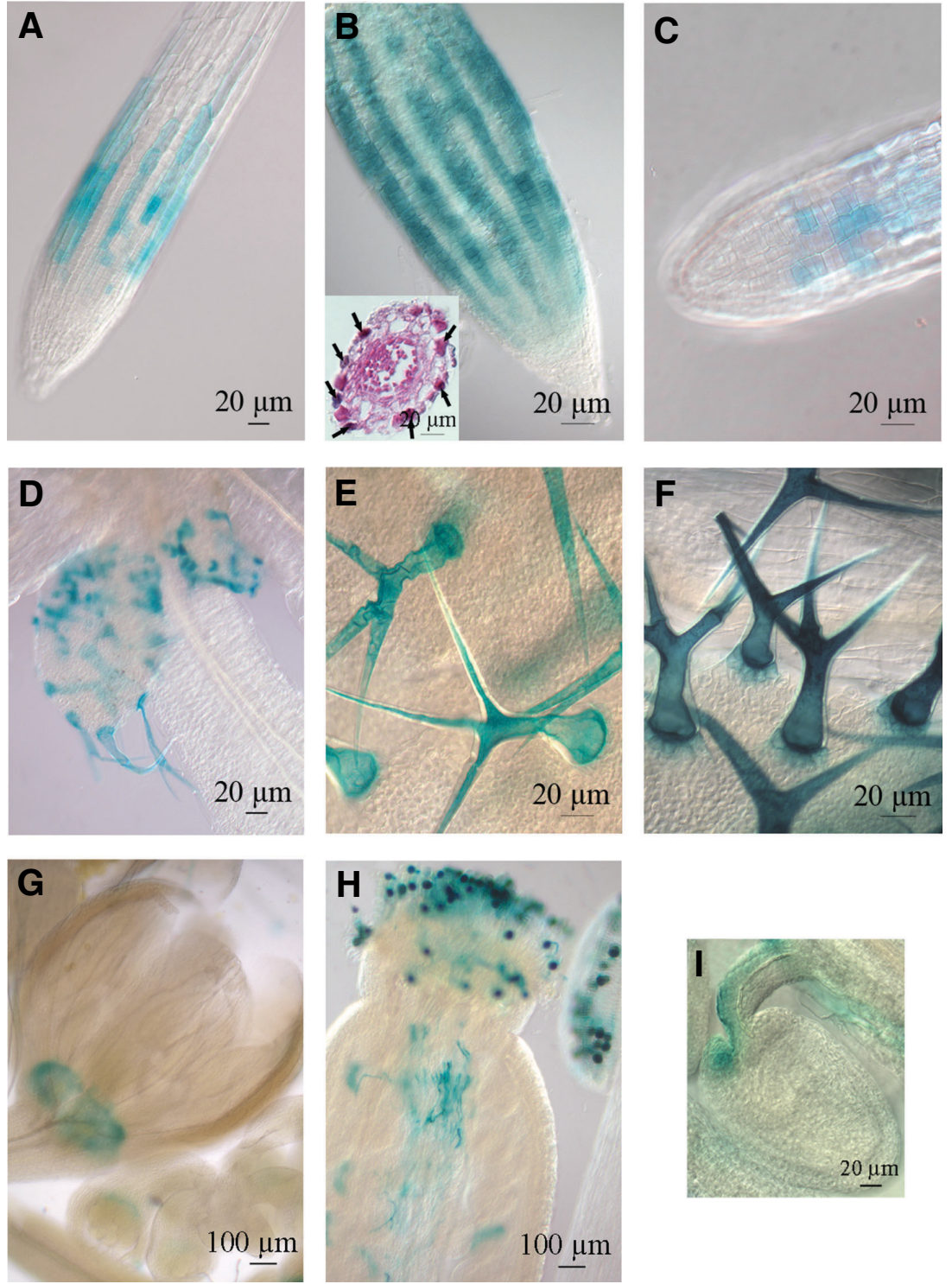

Fig. 3. AtSGP2 promoter activity in Arabidopsis thaliana lines. Differential GUS staining in the root epidermis in two different 10-day-old $T_{2}$ lines $(\mathbf{A}, \mathbf{B})$ and in the scm2 mutant background $(\mathbf{C})$. Insert in $(B)$ represents a transverse section in the elongation zone of the primary root: arrows show atrichoblast cells. The AtSGP2 promoter is strongly activated during trichome development: (D) leaf primordium, (E) young leaf and (F) mature leaf. No expression is detected in the socket cells. GUS staining is highlighted in the nectaries from young flower buds (G), in the pollen tubes at the pollination stage (H) and during fertilization of the female gametes (I).

stage 6 / 7 according to Smyth et al. (1990), blue staining was found in the receptacle, possibly in nectaries, but not in anthers or ovaries (Fig. 3G). In further stages (stages 10/11), blue staining was weak (Fig. 4A). Thereafter in stages $12 / 15$, the level gradually increased as pollen matured. AtSGP2promoter activity reached a maximum in mature pollen grains at anthesis (Fig. 4A). In order to establish the precise stage of AtSGP2promoter activity during male gametogenesis, the nuclei were stained with Hœchst in parallel to the GUS staining. At the uninucleated stage, microspores did not exhibit GUS activity (Fig. 4 B,F,G). After the first asymmetric mitosis (PMI), GUS activity remained undetectable in 
most of the young binucleated pollen grains (Fig. $4 \mathrm{C}, \mathrm{H}, \mathrm{I}$ ). At a later stage, a weak AtSGP2 promoter activity was seen in a few binucleated pollen grains (Fig. $4 \mathrm{D}, \mathrm{J}, \mathrm{K}$ ). After the second symmetric mitosis (PM II), the blue staining of the tricellular pollen showed a significantly increased intensity (Fig. $4 \mathrm{D}, \mathrm{L}, \mathrm{M})$, and it peaked in mature pollen at anthesis (Fig. 4 E,N,O).

Given the very strong expression in mature pollen, AtSGP2 expression was studies after pollination. After anther dehiscence, released blue stained pollen grains germinated on the stigma papillae (Fig. $3 \mathrm{H})$. It was easy to observe the deep blue staining at the distal end of the pollen tubes. Until ovule fertilization, the polarized growth of the pollen tube could be monitored by AtSGP2 promoter activity. The tips of the pollen tubes penetrate the papillae cuticle and continue to grow intrusively along the stigmatic papillae to the centre of the style into the transmitting tract towards the embryo sacs. AtSGP2 expression was observed in ovules immediately following fertilization (Fig. 3l).

\section{AtMAP3Kepsilon1 promoter activity marks some cells tar- geted by AtSGP}

Interaction between AtSGP1 and BnMAP3Kepsilon1 was previously shown using the yeast two hybrid technique (Champion et al., 2004). A $0.9 \mathrm{~Kb}$ fragment from the $5^{\prime}$ UTR of the AtMAP3Kepsilon 1 gene was cloned upstream of the GUS reporter gene. This region corresponds to the promoter region of the AtMYB5 gene (At3g13540), albeit in the reverse orientation. The AtMAP3Kepsilon 1 promoter activity was detected in leaf margins, in trichomes and in root apices of stably transformed $A$. thaliana lines (Fig. 5 $\mathrm{A}, \mathrm{B})$. The activity of this promoter was strongly detected in the root cap, both in columella stem cells and columella cells. This expression pattern overlapped with the AtSGP1 promoter activity in specific root cap cells and therefore the two proteins could interact thus being consistent with the previously observed protein-protein interaction.

A strong AtMAP3Kepsilon 1 promoter activity was also detected in

Fig. 4. AtSGP2 promoter activity during male gametogenesis. Histochemical localisation of GUS activity in transgenic lines. (A) Developing and opened flowers from stage 10 to stage 15 according to Smyth (1990). (B,C,D,E) Magnification of anthers at the different stages are detailed in (A). Microspore to pollen grain isolated from the different stages observed after GUS $(\mathbf{F}, \mathbf{H}, \mathbf{J}, \mathbf{L}, \mathbf{N})$ and Hochst $(\mathbf{G}, \mathbf{I}, \mathbf{K}, \mathbf{M}, \mathbf{O})$ staining. (F,G) Microspore. (H,I,J,K) Bicellular pollen. (L, $\mathbf{M}, \mathbf{N}, \mathbf{O})$ Tricellular pollen. Note that a light GUS staining is observed at the late bicellular stage $\mathbf{( J )}$. (N, $\mathbf{0})$ Mature tricellular pollen. Note the intense GUS staining at anthesis. VN: vegetative nucleus; GN: generative nucleus; SN: sperm cell nuclei. ovules, from early stages until ovule fertilization. In young ovules AtMAP3Kepsilon 1 promoter activity was noticed in the inner integuments (Fig. $5 \mathrm{C}$ ) that grow to enclose the nucellus. In the mature ovule, AtMAP3Kepsilon 1 promoter activity was detected in the inner and outer integuments, especially in the region surrounding the micropyle (Fig. 5D).

The AtMAP3Kepsilon 1 promoter activity was also examined in leaves where it was observed in trichome cells (Fig. 5B), identical to the AtSGP2promoter activity (Fig. 3 D,E,F). This co-expression suggests that AtSGP2 could potentially interact with AtMAP3Kepsilon 1 in planta, although no such interaction was detected in our yeast two hybrid studies.

\section{Discussion}

A fundamental question in biology is how pluripotent cells differentiate towards a specific cell phenotype. This question was addressed through a careful analysis of plant developmental stages using promoter.:GUSfusions constructs in various genetic backgrounds.

\section{Evolutionary features}

A comparison between the chromosome blocks including AtSGP1 and AtSGP2 reveals a duplicated segment containing a few genes, with limited synteny. Evidence for an ancient duplica-

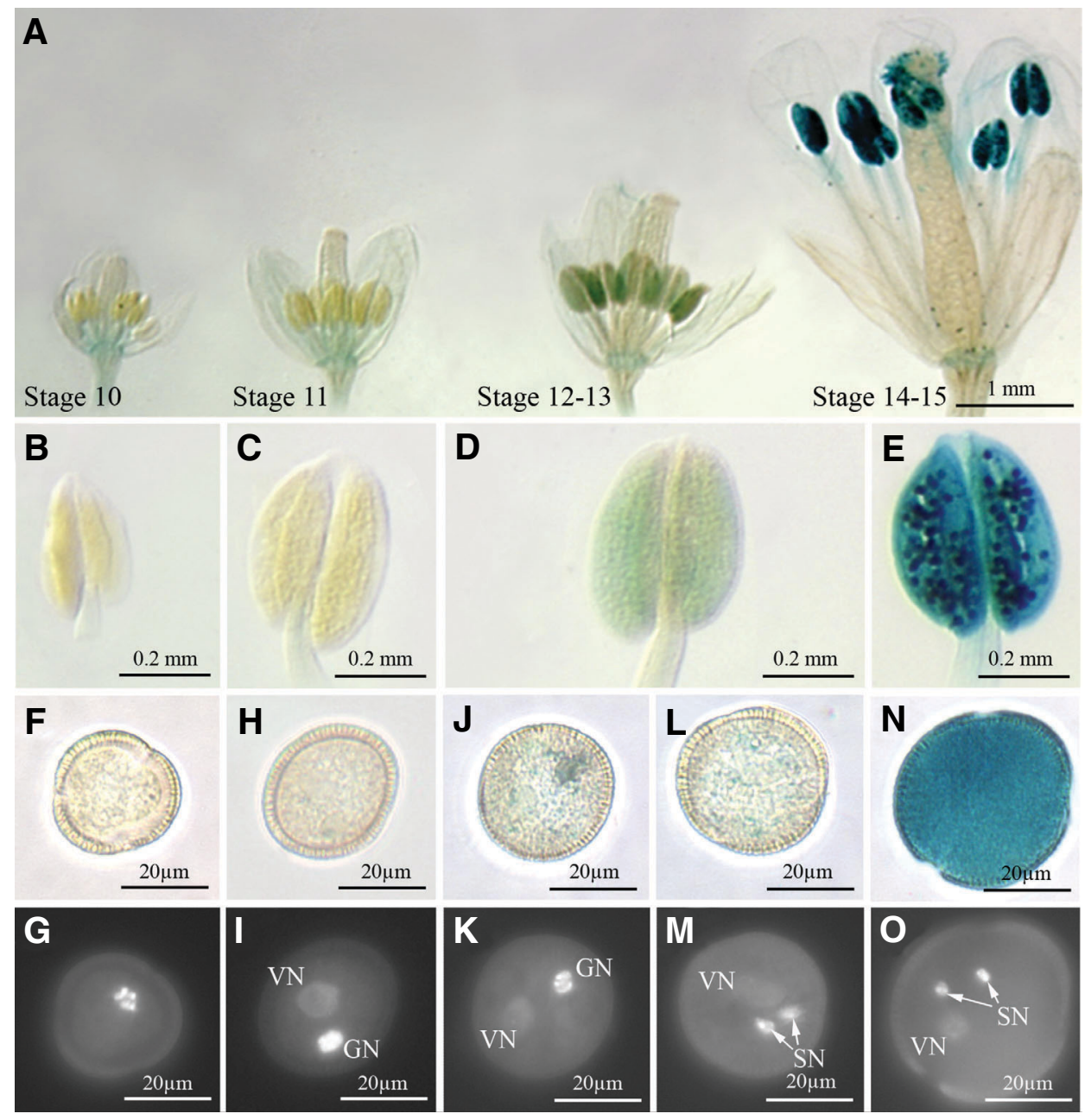


tion event is mostly provided by the number of synonymous substitutions per site, $\mathrm{Ks}=2.83$, indicative of a duplication event that pre-dates the Monocot-Eudicot divergence, 125 to 140 million years ago. Both genes and promoters have evolved since that time and the promoters of the paralogues AtSGP1 and AtSGP2 now drive activity in different cell types: stomata guard cells, QC, stele and trichomes, atrichoblasts, pollen grains, respectively. Our data provide evidence that these genes putatively play a common role in signalling cell differentiation in different specialized cell types. Our previous work proposed that the two paralogue genes, AtSGP1 and AtSGP2 were orthologues of S. pombe spg1 (Champion et al., 2004). However, their promoter activities reveal that AtSGP proteins do not link cell cycle exit to cytokinesis in plants. This reinforces the recent conclusions of Chaiwongsar et al. (2006) who demonstrated that AtMAP3Kepsilon function was not related to that of the core yeast component cdc7p. These proteins have evolved in plants to perform a function different from the SIN pathway (Bedhomme et al., 2008). Evolution seems to have recycled ancient signalling components to derive a new plant signalling pathway in Angiosperms.

\section{AtSGP2 promoter activity and cell fate specification}

The pattern of AtSGP2 promoter activity in root epidermal cell files is similar to that of the homeobox transcription factor GLABRA2 (Masucci et al., 1996). Indeed, cytological observations localised the AtSGP2 expression in atrichoblasts (non-hair root epidermal cells), in accordance with the gene expression map of the $A$. thaliana root by Birnbaum et al. (2003). Root epidermal cells differentiate into two cell types, namely root-hair cells (trichoblasts) and hairless cells (atrichoblasts) in a position-dependent pattern. The trichoblasts lie over the junction of two cortical cells, whereas the atrichoblasts overlie a single cortical cell (Berger et al., 1998a,b; Dolan et al., 1993; Galway et al., 1994). Furthermore, atrichoblast cells exit the mitotic cycle and are directed towards terminal differentiation earlier than their neighbours (Schiefelbein, 2003). For epidermis development, the non functional membrane-bound receptor kinase Scrambled (SCM) (Kwak et al., 2005) enables epidermal cells to perceive positional cues (Llompart et al., 2003). The SCM gene is required for proper positiondependent cell-type patterning. We observed that the AtSGP2 promoter activity pattern in the root epidermis of the $s c m-2$ mutant was identical to the pattern of GLABRA2in the scm-2mutant. This led us to postulate that $S C M$ is epistatic to AtSGP2, both participating in a putative signalling pathway that acts early to promote position-dependent cell fate specification of non hair cells.

A similar set of proteins controls the patterning mechanism of hairs in the leaf epidermis (trichomes), of non hair cells in the root epidermis (atrichoblasts), of stomata in the hypocotyl epidermis (not detailed here) and of seed coat cells (pigments and mucilage) (Pesch and Hulskamp, 2004; Schiefelbein, 2003; Zhang et al., 2003). AtSGP2 promoter activity suggests its involvement in trichome cell fate specification. Similarly the AtMAP3Kepsilon 1 promoter shows activity in trichome cells. This co-expression with AtSGP2suggests that both proteins could interact in planta. It is interesting to note that $A T S G P 1$ promoter activity is detected in stomata guard cells, whereas AtSGP2 and AtMAP3Kepsilon 1 promoter activities were observed in trichomes. Results from Glover et al. (1998) indicated that in the leaf epidermis, the cell differentiation programs for stomata and trichomes arise from the same pool of uncommitted cells.

Furthermore, AtMYB5 promoter-driven GUS activity was previously detected by Li et al. (1996) in leaf trichomes and in the seed coat of the young seed. These observations are identical to those reported in this work for the AtMAP3Kepsilon 1 promoter (which consists of the same genome region but in the reverse orientation). This provides evidence that both genes are involved in trichome and seed coat cell fate specification.

\section{AtSGPs promoter activity and maintenance of cell differen- tiation}

GFP driven by the synthetic $D R 5$ promoter co-localized with AtSGP1 promoter activity, suggesting that AtSGP1 is mainly expressed in the $Q C$. This conclusion agrees with the transcriptome analyses of Birnbaum et al. (2003) and Brady et al. (2007). The $\mathrm{QC}$ is an organizing centre which maintains the stem cell function of initials (van den Berg etal., 1997). In contrast, cells from the QC display a reduced mitotic activity. PLT1 and PLT2 genes are redundantly required for the specification of organizing $Q C$ cells and for the maintenance of root stem cells. In plt1 plt2 double
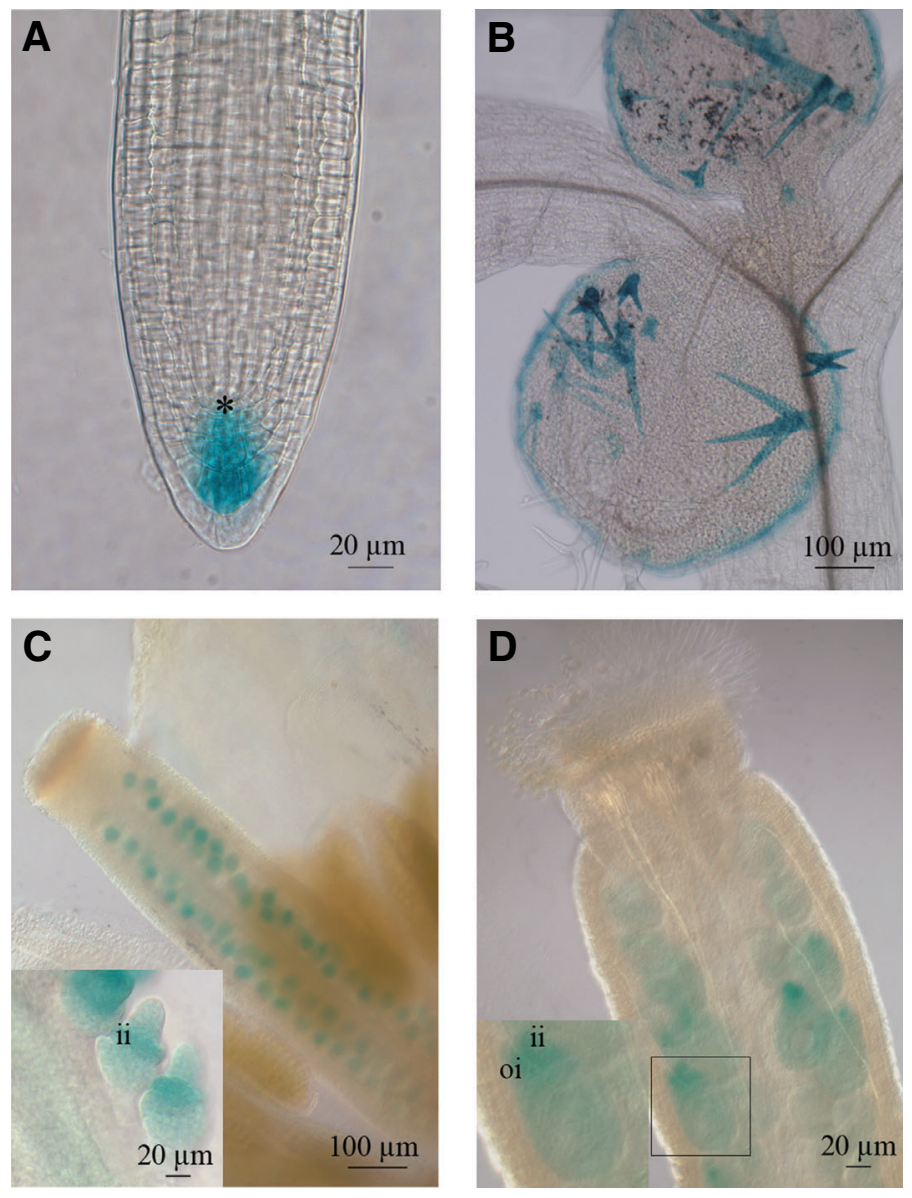

Fig. 5. Arabidopsis thaliana AtMAP3Kepsilon 1 promoter activity in transgenic $\mathbf{T}_{\mathbf{2}}$ lines. Collumella cells at the root tip (A). QC is marked by an asterisk. Trichomes from the young leaf epidermis (B). (C) Carpels with young developing ovules. Insert in (C) represents a detailed view of the same ovule stage; ii: inner integument. (D) In mature ovules, the GUS staining is observed in the micropyle region as shown in the insert; ii: inner integument and oi: outer integument. 
mutants, QC and stem cell identity are lost (Aida et al., 2004) and AtSGP1 promoter activity was seen to spread throughout additional layers of differentiated cells, proximal to the mis-specified QC cells. Therefore a functional $Q C$ was not necessary for AtSGP1 promoter activity. This suggests that PLT does not genetically interact with AtSGP1. Nevertheless, PLTexpression is required to restrict $A t S G P 1$ promoter activity to the QC, meaning that, genetically, $P L T$ is upstream of $A T S G P 1$. The wide distribution of AtSGP1 in the root cap of plt1 plt2 double mutants confirms that AtSGP1 also specifies the differentiated collumella cells. Interestingly, our results show an overlap between the promoter activity profiles of AtSGP1 and AtMAP3Kepsilon 1 in the columella cells. This co-expression indicates that they could be protein partners in planta. Their physical interaction was previously detected in yeast double hybrid studies (Champion et al., 2004).

We also found AtSGP1 promoter activity in the stele, particularly at stages that include differentiated cells. Again, such observations agree with the recent root transcriptome map of Brady et al. (2007). Our data set suggests that AtSGP1 could also be involved in specification and maintenance of stele cell differentiation.

The asymmetric division of a leaf epidermal cell produces a primary meristemoid and a pavement cell. The meristemoid undergoes one, two or three asymmetric mitosis before guard mother cell specification (Donnelly et al., 1999). The last mitosis of the guard mother cell is symmetric and produces the two guard cells (Larkin et al., 1997). AtSGP1 promoter activity was only detected in mature stomata guard cells. AtSGP1promoter activity in these cells suggests that AtSGP1 has a role in determining the function of these differentiated cells.

Finally, we noticed that the AtSGP2promoter activity was very strong in mature pollen. In $A$. thaliana, asymmetric division of a uninucleated microspore in pollen mitosis I (PMI) produces a large vegetative cell and a small generative cell, each having a different fate. Whereas the vegetative cell does not further divide, the generative cell divides symmetrically to produce two sperm cells. The AtSGP2promoter become active after the asymmetric division of the male gametophyte, and it continues to be active during pollen tube growth into the embryo sac. This suggests that AtSGP2 promoter activity is required for the determination of pollen function. The AtSGP2 promoter activity in male gametophytes observed in this work is in full accordance with the transcriptome analyses from Pina et al. (2005) and Honys and Twell (2004). About 500 A. thaliana genes exhibit a pollen expression profile identical to AtSGP2, most of them with a reduced level. The promoter activity during pollen tube growth should be very useful to check for pollination, fertilisation and selfincompatibility response.

Moreover, ATSGP genes could play a role either in early mechanisms in cell fate determination (atrichoblasts, integuments, trichomes) or later in the determination of cell function (QC, stomata guard cells, pollen, stele). This suggests that AtSGP1 and ATSGP2promoters can be used as cellular markers of early and late events in cell differentiation. Our data should be used as a starting point towards genetic and functional analyses. We are presently designing experiments to test the hypothesis that the two paralogous GTPase genes, AtSGP1 and AtSGP2, are signalling components involved in mechanisms of cell differ- entiation.

Studies in Mammalian systems have revealed that pluripotency is controlled by a regulatory core of transcription factors that activate genes critical for self renewal of stem cells and repress genes initiating differentiation (Sun et al., 2006; Wang et al., 2006). Similar data from plant cells and mammalian cells suggest that stem cells can be specified by kingdom specific patterning mechanisms, connected to related epigenetic stem cell factors (Ben Scheres, 2007). What component combinations signal and confer differentiation to plant specific cell types remain to be elucidated.

\section{Materials \& Methods}

\section{Plant material and transformation}

Constructions of AtSGP1 and AtSGP2 promoter-GUS gene fusions were introduced into Agrobacterium tumefaciens (HBA105 and Agl1 respectively). Arabidopsis thaliana plants (Columbia ecotype or mutant lines) were transformed by the floral dip method as described (Clough and Bent, 1998). The progeny of the $\mathrm{T}_{0}$ plants was sown on a $0.5 \mathrm{X}$ Murashige and Skoog (MS) medium, complemented with kanamycine $(50 \mathrm{mg} / \mathrm{mL})$. After 2 weeks, a fraction of the resistant $T_{1}$ plantlets were tested for GUS staining while the remaining plants were transferred to soil in a greenhouse under short-day conditions for 2 weeks, then under long-day conditions. The $T_{1}$ and $T_{2}$ generations were selected on kanamycin.

\section{AtSGP1, AtSGP2 and AtMAP3Kepsilon1 promoter::GUS fusion con- structs}

Promoter regions were amplified from Arabidopsis thaliana genomic DNA (Columbia ecotype) by PCR. Using primers containing added restriction sites, a $2.2 \mathrm{~kb}$ region upstream from the ATG start codon was chosen for AtSGP1 (At5g54849) and a $1.2 \mathrm{~kb}$ region for AtSGP2 (At3g21700). The AtSGP1 primers were:

G1proEcoPst 5'-CGGAATTCTGCAGGGGCGGATACATGAATAAAC, and G1proBam 5'-CGGGATCCTGATGAAACAGAGAGAAAGTTATGTG. The AtSGP2 primers were:

G2proEcoR1 5'-CAGAATTCGCCAATGATGGTGAAAGATGTAG, and G2proSal1 5'-ACGTCGACTGAGAAAATTCAAAATTCGAAATC. The AtSGP1 promoter was introduced into the PTAK plasmid, upstream of the $\beta$-glucuronidase (GUS) gene as a Pst1/BamH1 fragment. The AtSGP2 promoter PCR fragment was ligated into pGEM-T (Promega, Madison, $\mathrm{WI})$. The DNA sequence of both cloned promoter region sequences was verified before subsequent cloning. For the AtSGP1 promoter, a $1.2 \mathrm{~kb}$ promoter region plus the GUS gene was excised from the construct in pTAK as a HindIII/EcoRI fragment and subcloned into the pCW83 binary plasmid. The promoter fragment of AtSGP2obtained by digestion (EcoRI/ Sall) was transferred into the pPR97 binary vector upstream of the GUS gene. As a result for both AtSGP1 and AtSGP2, a $1.2 \mathrm{~kb}$ promoter region was used for subsequent promoter activity analyses. For AtMAP3Kepsilon 1 ( $A t 3 g 13539$ a $0.9 \mathrm{~kb}$ region upstream of the ATG start codon was chosen. The AtMAP3Kepsilon 1 gene is localised upstream of the AtMYB5gene in the reverse orientation (Jouannic et al., 2001). This means that the AtMAP3Kepsilon 1 promoter region is also the promoter of the At3g13540 (MYB5) gene, and this has been previously cloned (Li et al., 1996). The primers used for PCR amplification were: 3Ke1proEcoR1 5'CAGAATTCTCTTTCCACTAGGGTTTCGTG, and 3Ke1proSal1 5'ACGTCGAC CTCTTCTCGTTTCTTCTTCCTCC. The promoter fragment of AtMAP3Kepsilon 1 was cloned as described for the AtSGP2promoter. The final constructs were transferred into Agrobacteriumby electroporation.

\section{Histochemical GUS and Hœchst staining}

Histochemical localization of GUS activity was performed on 10- or 12day-old $T_{1}$ and $T_{2}$ generation seedlings and on inflorescences from $T_{1}$ and $\mathrm{T}_{2}$ plants at various stages of floral development (unopened bud to mature 
silique). Samples (from at least 10 different lines) were fixed in cold $90 \%$ acetone for $30 \mathrm{~min}$. They were washed with staining buffer $(0.5 \mathrm{mM}$ sodium phosphate buffer, $5 \mathrm{mM}$ ferrocyanide, $5 \mathrm{mM}$ ferricyanide) and placed in X-Gluc (5-bromo-4-chloro-3-indolyl- $\beta$-D-glucuronide) staining solution (staining buffer containing $39 \mathrm{mM} \mathrm{X-Gluc} \mathrm{from} \mathrm{a} 767 \mathrm{mM}$ stock solution in DMSO). Tissues were vacuum-infiltrated for $10 \mathrm{~min}$ and incubated at $37^{\circ} \mathrm{C}$ for $1.5 \mathrm{~h}$. Finally the GUS buffer was replaced by 30 $\%$ ethanol during $20 \mathrm{mins}, 50 \%$ ethanol during $20 \mathrm{~min}$ and $70 \%$ ethanol for $20 \mathrm{~min}$, then left overnight to destain the tissues prior to observation. Before microscope observation, ethanol was removed and samples were cleared by an overnight incubation in chloral hydrate solution (chloral hydrate / glycerol / water 8:2:1(w/v/v)).

For pollen nuclei visualization, samples were fixed in ethanol:acetic acid (3:1) for 10 min just after flower GUS staining and rehydrated in and ethanol series $(70 \%, 50 \%, 30 \%)$ and finally water. Pollen walls were digested by incubation in a solution containing $0.3 \%$ pectolyase, $0.3 \%$ cytolyase and $0.3 \%$ cellulose for $30 \mathrm{~min}$ at $37{ }^{\circ} \mathrm{C}$. Enzymes were eliminated by 3 washes in water and DNA was stained with $5 \mu \mathrm{g} / \mathrm{mL}$ Hœchst in $1 \%$ Triton X100 and visualised under UV light.

For sectioning, GUS-stained root segments were fixed in $3 \%$ glutaraldehyde diluted in PBS 1X (pH 7.2) and washed 4 times in 1X PBS ( $\mathrm{pH} 7.2)$ for $10 \mathrm{~min}$. Then samples were dehydrated successively in ethanol (,30\%; $50 \%, 70 \%, 80 \%, 95 \%$ and $100 \%$ ) with a 15 min incubation at each concentration. The plantlets were soaked in $1 / 3$ histoclear $2 / 3$ ethanol 100 $\%$ for $15 \mathrm{~min}, 1 / 2$ histoclear $1 / 2$ ethanol $100 \%$ with eosine for $15 \mathrm{~min}$, histoclear pure for $15 \mathrm{~min}$ and overnight in $1 / 2$ histoclear $\left(60{ }^{\circ} \mathrm{C}\right) 1 / 2$ ParaplastPlus ${ }^{\circ}\left(60^{\circ} \mathrm{C}\right)$. The next day, roots were embedded in Paraplast Plus $\AA$ and cut into $8 \mu \mathrm{m}$-thick sections using a microtome.

Starch granules were visualised in the collumella as described by Willemsen et al. (1998).

\section{Light and confocal microscopy}

Images of Arabidopsis thaliana roots and inflorescences stained in XGluc solution were captured on a Zeiss Axioskop Imaging microscope equipped with a Sony Power HAD camera (Paris, France) and the Axiovision 1.01 (Zeiss, Jena, Germany) software, and processed using Adobe Photoshop 6 software.

Roots of the DR5::GFP line were observed using a confocal microscope (Leica SP2). For GFP detection, excitation was performed at 488 $\mathrm{nm}$, with fluorescence emission captured between 510 and $514 \mathrm{~nm}$.

\section{Acknowledgements}

We would like to thank $B$. Scheres (University of Utrecht) for providing plt1-4 plt2-2 double mutant seeds, J. Schiefelbein (University of Michigan) for scm-2 mutant seeds and C. Perrot Rechenmann (ISV, Gif sur $Y$ vette) for DR5:: GFP seeds. We acknowledge the assistance of R. Boyer (IBP, Orsay) and $S$. Domenichini (IBP, Orsay) for the figures. We are especially grateful to N. Glab (IBP, Orsay) for advice and helpful discussions and to $G$. Noctor and M. Hodges (IBP, Orsay) for improving the manuscript. A. Pulido received a post-doctoral fellowship from the Spanish MEC.

\section{References}

AIDA M, BEISD, HEIDSTRA R, WILLEMSEN V, BLILOUI, GALINHAC, NUSSAUME L, NOHYS, AMASINO R, SCHERES B. (2004). The PLETHORA genes mediate patterning of the Arabidopsis root stem cell niche. Cel/119: 109-20.

BEDHOMME M, JOUANNIC S, SIMANIS V, HENRY Y. (2008). Plants, MEN and SIN. Plant Physiol Biochem 46: 1-10

SCHERES B. (2007). Stem-cell niches: nursery rhymes across kingdoms. Nat Rev Mol Cell Bio/8: 345-354.

BERGER F, HASELOFF J, SCHIEFELBEIN J, DOLAN L. (1998A). Positional information in root epidermis is defined during embryogenesis and acts in domains with strict boundaries. Curr Bio/8: 421-30.

BERGER F, HUNG CY, DOLAN L, SCHIEFELBEIN J (1998B). Control of cell division in the root epidermis of Arabidopsis thaliana. Dev Bio/194: 235-45.

BIRNBAUM K, SHASHA DE, WANG JY, JUNG JW, LAMBERT GM, GALBRAITH DW, BENFEY PN. (2003). A gene expression map of the Arabidopsis root. Science 302: 1956-60.

BRADY SM, ORLANDO DA, LEE JY, WANG JY, KOCH J, DINNENY JR, MACE D, OHLER U, BENFEY PN. (2007). A high-resolution root spatio temporal map reveals dominant expression patterns. Science 318: 801-806.

CHAIWONGSAR S. OTEGUI MS, JESTER PJ, MONSON SS, KRYSAN PJ. (2006). The protein kinase genes MAP3K epsilon 1 and MAP3K epsilon 2 are required for pollen viability in Arabidopsis thaliana. Plant J 48: 193-205.

CHAMPION A, JOUANNIC S, GUILLON S, MOCKAITIS K, KRAPP A, PICAUD A, SIMANIS V, KREIS M, HENRY Y. (2004). AtSGP1, AtSGP2 and MAP4K alpha are nucleolar plant proteins that can complement fission yeast mutants lacking a functional SIN pathway. J Cell Sci117: 4265-75.

CLOUGH SJ, BENT AF. (1998). Floral dip: a simplified method for Agrobacteriummediated transformation of Arabidopsis thaliana. Plant J 16: 735-43.

DOLAN L. (2006). Positional information and mobile transcriptional regulators determine cell pattern in the Arabidopsis root epidermis. J Exp Bot 57: 51-4.

DOLAN L, JANMAAT K, WILLEMSEN V, LINSTEAD P, POETHIG S, ROBERTS K, SCHERES B. (1993). Cellular organisation of the Arabidopsis thaliana root. Development 119: 71-84.

DONNELLY PM, BONETTA D, TSUKAYA H, DENGLER RE, DENGLER NG. (1999). Cell cycling and cell enlargement in developing leaves of Arabidopsis. Dev Bio/215: 407-19.

GALWAY ME, MASUCCI JD, LLOYD AM, WALBOT V, DAVIS RW, SCHIEFELBEIN JW. (1994). The TTG gene is required to specify epidermal cell fate and cell patterning in the Arabidopsis root. Dev Bio/ 166: 740-54.

GLOVER BJ, PEREZ-RODRIGUEZ M, MARTIN C. (1998). Development of several epidermal cell types can be specified by the same MYB-related plant transcription factor. Development 125: 3497-508.

HONYS D, TWELL D. (2004). Transcriptome analysis of haploid male gametophyte development in Arabidopsis. Genome Bio/5: R85.

JOUANNIC S, CHAMPION A, SEGUI-SIMARRO JM, SALIMOVA E, PICAUD A, TREGEAR J. TESTILLANO P, RISUENO MC, SIMANIS V, KREIS $M$ et al (2001). The protein kinases AtMAP3Kepsilon1 and BnMAP3Kepsilon1 are functional homologues of $\mathrm{S}$. pombe cdc7p and may be involved in cell division Plant J 26: 637-49.

KWAK SH, SHEN R, SCHIEFELBEIN J. (2005). Positional signaling mediated by a receptor-like kinase in Arabidopsis. Science 307: 1111-3.

LARKIN JC, MARKS MD, NADEAU J, SACK F. (1997). Epidermal cell fate and patterning in leaves. Plant Cel/9: 1109-20.

LI, S. F., SANTINI, J. M., NICOLAOU, O. AND PARISH, R. W. (1996). A novel mybrelated gene from Arabidopsis thaliana. FEBS Lett 379, 117-21.

LLOMPART B, CASTELLS E, RIO A, ROCA R, FERRANDO A, STIEFEL V, PUIGDOMENECH P, CASACUBERTA JM. (2003). The direct activation of MIK a germinal center kinase (GCK)-like kinase, by MARK, a maize atypical receptor kinase, suggests a new mechanism for signaling through kinase-dead receptors. J Biol Chem 278: 48105-11.

MACALISTER CA, OHASHI-ITO K, BERGMANN DC. (2007). Transcription factor control of asymmetric cell divisions that establish the stomatal lineage. Nature 445: 537-40.

MASUCCI JD, RERIE WG, FOREMAN DR, ZHANG M, GALWAY ME, MARKS MD, SCHIEFELBEIN JW. (1996). The homeobox gene GLABRA2 is required for position-dependent cell differentiation in the root epidermis of Arabidopsis thaliana. Development 122: 1253-60.

PESCH M, HULSKAMP M. (2004). Creating a two-dimensional pattern de novo during Arabidopsis trichome and root hair initiation. Curr Opin Genet Dev 14: 422-7.

PINA C, PINTO F, FEIJO JA, BECKER JD. (2005). Gene family analysis of the Arabidopsis pollen transcriptome reveals biological implications for cell growth, division control, and gene expression regulation. Plant Physio/ 138: 744-56.

SCHIEFELBEIN J. (2003). Cell-fate specification in the epidermis: a common patterning mechanism in the root and shoot. Curr Opin Plant Bio/6: 74-8.

SCHMIDT S, SOHRMANN M, HOFMANN K, WOOLLARD A, SIMANIS V. (1997) The Spg1p GTPase is an essential, dosage-dependent inducer of septum 
formation in Schizosaccharomyces pombe. Genes Dev11: 1519-34.

SIMANIS V. (2003). The mitotic exit and septation initiation networks. JCel/Sci116: 4261-2.

SMYTH DR, BOWMAN JL, MEYEROWITZ EM. (1990). Early flower development in Arabidopsis. Plant Cel/2: 755-67.

SUN Y, LI H, YANG H, RAO MS, ZHAN M. (2006). Mechanisms controlling embryonic stem cell self renewal and differentiation. Crit Rev Eukaryot Gene Express 16: 311-231

ULMASOV T, MURFETT J, HAGEN G, GUILFOYLE TJ. (1997). Aux/IAA proteins repress expression of reporter genes containing natural and highly active synthetic auxin response elements. Plant Cel/9: 1963-71.
VAN DEN BERG C, WILLEMSEN V, HENDRIKS G, WEISBEEK P, SCHERES B (1997). Short-range control of cell differentiation in the Arabidopsis root meristem. Nature 390: 287-9.

WANG J, RAO S, CHU J, SHEN X, LEVASSEUR DN, THEUNISSEN TW, ORKIN $\mathrm{SH}$. (2006). A protein interaction network for pluripotency of embryogenic stem cells. Nature 444: 364-368.

WILLEMSEN V, WOLKENFELT H, DE VRIEZE G, WEISBEEK P, SCHERES B (1998). The HOBBIT gene is required for formation of the root meristem in the Arabidopsis embryo. Development 125: 521-31.

ZHANG F, GONZALEZ A, ZHAO M, PAYNE CT, LLOYD A. (2003). A network of redundant bHLH proteins functions in all TTG1-dependent pathways of Arabidopsis. Development 130: 4859-69.

\section{Further Related Reading, published previously in the Int. J. Dev. Biol.}

See our recent Special Issue Fertilization, in honor of David L. Garbers and edited by Paul M. Wassarman and Victor D. Vacquier at: http://www.ijdb.ehu.es/web/contents.php?vol=52\&issue=5-6

See our recent Special Issue Plant Development, edited by José Luis Micol and Miguel A. Blázquez at:

http://www.ijdb.ehu.es/web/contents.php?vol=49\&issue=5-6

Epidermal differentiation: trichomes in Arabidopsis as a model system

Swen Schellmann and Martin Hülskamp

Int. J. Dev. Biol. (2005) 49: 579-584

Ethylene induces stomata differentiation in Arabidopsis.

L Serna and C Fenoll

Int. J. Dev. Biol. (1996) 40: S123-S124

Balance between cell division and differentiation during plant development

Elena Ramirez-Parra, Bénédicte Desvoyes and Crisanto

Gutierrez

Int. J. Dev. Biol. (2005) 49: 467-477

Mechanisms of the proliferation and differentiation of plant cells in cell culture systems.

H Fukuda, M Ito, M Sugiyama and A Komamine

Int. J. Dev. Biol. (1994) 38: 287-299

Flower and fruit development in Arabidopsis thaliana

Pedro Robles and Soraya Pelaz

Int. J. Dev. Biol. (2005) 49: 633-643

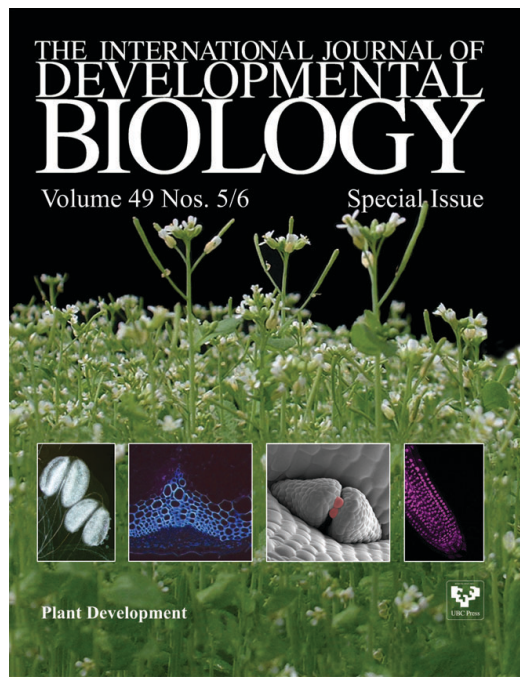

2006 ISI **Impact Factor $=3.577^{* *}$

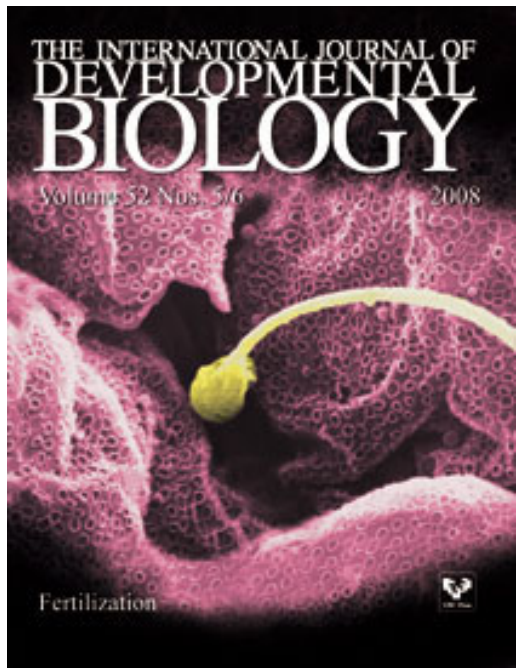

